\title{
Ultrafast Spectroscopy And Computational Study Of The Photochemistry Of \\ Diphenylphosphoryl Azide: Direct Spectroscopic Observation Of A Singlet
}

\section{Phosphorylnitrene}

\author{
Shubham Vyas* \\ Department of Chemistry, The Ohio State University, 100 West $18^{\text {th }}$ Avenue, Columbus, Ohio
}

\section{Abstract}

In order to characterize the active site of human paraoxonase 1 (HuPON1), photochemistry of a possible photoaffinity label diphenylphosphoryl azide was studied by femtosecond transient absorption spectroscopy, chemical analysis of light-induced reaction products as well as by RI-CC2/TZVP and TD-B3LYP/TZVP computational methods. Theoretical methods predicted two possible mechanisms for singlet diphenylphosphorylnitrene formation from the photoexcited phosphoryl azide. (i) Energy transfer from the $\left(\pi, \pi^{*}\right)$ singlet excited state, localized on a phenyl ring, to the azide moiety, thereby leading to the formation of the singlet excited azide, which subsequently loses molecular nitrogen to form the singlet diphenylphosphorylnitrene. (ii) Direct irradiation of the azide moiety to form an excited singlet state of the azide, which in turn loses molecular nitrogen to form the singlet diphenylphosphorylnitrene. Two transient species were observed upon ultrafast photolysis (260 $\mathrm{nm}$ ) of diphenylphosphoryl azide. The first transient absorption, centered at $475 \mathrm{~nm}$ (lifetime $(\tau)$ $\sim 28 \mathrm{ps}$ ), was assigned to a $\left(\pi, \pi^{*}\right)$ singlet $\mathrm{S}_{1}$ excited state localized on a phenyl ring, and the second transient observed at $525 \mathrm{~nm}(\tau \sim 480$ ps $)$ was assigned to singlet

\footnotetext{
* All of the efforts, contributions and guidance provided by Dr. Sivaramakrishnan Muthukrishnan, Dr. Jacek Kubicki, Prof. Matthew S. Platz and Prof. Christopher M. Hadad to complete this work are gratefully acknowledged.
} 
diphenylphosphorylnitrene. Experimental and computational results obtained from the study of diphenylphosphoramidate, along with the results obtained with diphenylphosphoryl azide, supported the mechanism of energy transfer from the singlet excited phenyl ring to the azide moiety, followed by nitrogen extrusion to form the singlet phosphorylnitrene. Ultrafast timeresolved studies performed on diphenylphosphoryl azide with the singlet nitrene quencher, tris(trimethylsilyl)silane, confirmed the spectroscopic assignment of singlet diphenylphosphorylnitrene to the $525 \mathrm{~nm}$ absorption band.

Keywords: Phosphorylnitrene, ultrafast spectroscopy, RI-CC2, TD-B3LYP, energy transfer

\section{Introduction}

Several organophosphorus (OP) compounds (Scheme 1) are chemical warfare agents, which are potential threats to human society. ${ }^{1}$ These chemical agents inhibit acetylcholinesterase (AChE) in the human body, and this efficient enzyme is a serine hydrolase which is responsible for the hydrolysis of the neurotransmitter, acetylcholine. ${ }^{2}$ Two different approaches have been used in order to protect humans from OP exposure: (a) post-exposure treatment with powerful nucleophiles, such as pyridinium oximes, ${ }^{3}$ that can reactivate AChE; or (b) a pre-exposure with a prophylactic enzyme (bioscavenger) ${ }^{4}$ that either catalytically hydrolyses the lethal OP compounds or at least competes with $\mathrm{AChE}$ for reaction with the OP. The latter method is of obvious advantage due to the short time available for first-responders to treat patients after an OP exposure.

Recently, ${ }^{5}$ human paraoxonase 1 (HuPON1) was recognized as a potential catalytic bioscavenger to hydrolyze OPs prior to the inhibition of AChE. HuPON1 is a natural human enzyme, which is normally associated with high-density lipoprotein (HDL), and this enzyme can catalyze the hydrolysis of some OP compounds. However, the catalytic efficiency of HuPON1 is 
not sufficient to offer complete protection against OP nerve agents. ${ }^{5}$ Since, the exact mechanism of OP hydrolysis by HuPON1 is not completely understood, an efficient, catalytic bioscavenger for OPs has not yet been developed. With the goal of improving the catalytic efficiency, several mutants $^{6}$ of PON1 have been prepared, albeit with little success. Thus, a more detailed understanding of the critical active site residues will aid in the design of mutants with the desired catalytic properties.

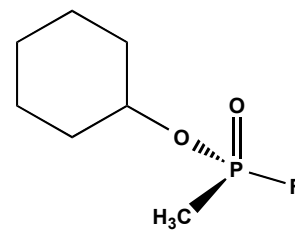

Cyclosarin

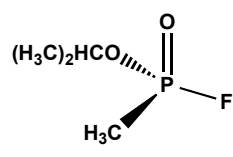

Sarin

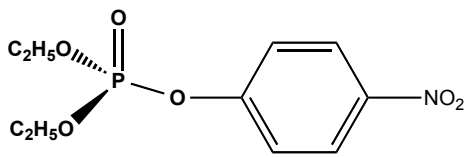

DPP-N 3
Paraoxon

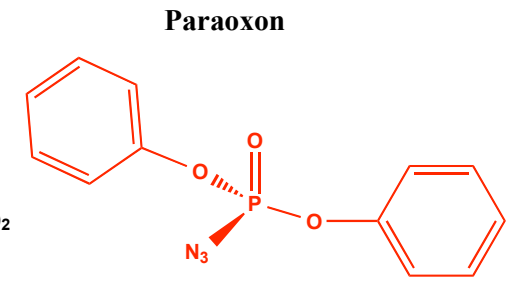

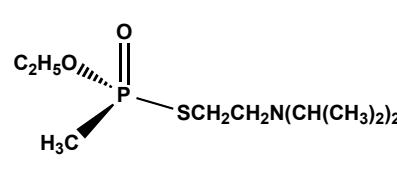

VX

Scheme 1. Some organophosphorus (OP) nerve-agents (in black) and photoaffinity label DPP-N 3 (in red)

A commonly employed method used to obtain structural information about an active site is photoaffinity labeling. ${ }^{7}$ An ideal photoaffinity label (PAL) is similar in structure to a substrate of an enzyme and has a strong affinity to bind at the active site of the enzyme. However, the PAL contains a photoreactive group, which upon irradiation produces a highly reactive intermediate that can randomly and rapidly insert into the amino acids present in the vicinity of the binding site. Following the PAL insertion, mass spectrometric and proteomic analysis of the PAL-enzyme complex provides important structural information about the active site. Since many OP compounds are substrates with PON1, we designed a structurally similar PAL. Diphenylphosphoryl azide (DPP- $\mathrm{N}_{3}$ ) is an OP compound in which one of the bonds at the 
phosphorus center is replaced with an azide group as shown in Scheme 1. It is known that upon photolysis, phosphoryl azides can generate phosphorylnitrenes, which can insert into unactivated C-H bonds. ${ }^{8,9,10}$ Subsequently, the photochemistry of DPP-N $\mathrm{N}_{3}$ was studied by laser flash photolysis (LFP) and electron paramagnetic resonance (EPR) methods. ${ }^{11,12}$ Although triplet phosphorylnitrene has been detected by Gohar and Platz ${ }^{12}$ using nanosecond LFP techniques, the shorter lived reactive singlet ( $\left.{ }^{1} \mathrm{DPP}-\mathrm{N}\right)$ analogue has not been spectroscopically observed. The lifetime of triplet diphenylphosphorylnitrene $\left({ }^{3} \mathrm{DPP}-\mathrm{N}\right)$ is on the order of microseconds in various solvents, while the lifetime of singlet diphenylphosphorylnitrene ( $\left.{ }^{1} \mathrm{DPP}-\mathrm{N}\right)$, which was not observed, was estimated to be on the order of 1 nanosecond (ns) in 1,2-dichloroethane and methanol. Hence, confirming the formation of ${ }^{1} \mathrm{DPP}-\mathrm{N}$ and directly measuring its lifetime would be a significant step towards the development of a PAL for PON1.

Earlier, we reported a computational investigation concerning the potential of phosphoryl azides to function as precursors to phosphorylnitrenes. ${ }^{13}$ Herein, we report the first experimental observation of a singlet phosphorylnitrene. We investigated the photochemistry of DPP- $\mathrm{N}_{3}$ by computational approaches, chemical analysis of light-induced products (in acetonitrile and cyclohexane), and femtosecond ultrafast UV-Vis transient absorption spectroscopic methods in acetonitrile. Computational studies predicted the formation of ${ }^{1} \mathrm{DPP}-\mathrm{N}$ via two plausible mechanisms, namely: (a) energy transfer that can occur from the $\left(\pi, \pi^{*}\right) \mathrm{S}_{1}$ excited state of the phenyl rings to the azide group, which forms ${ }^{1} \mathrm{DPP}-\mathrm{N}$ and an $\mathrm{N}_{2}$ molecule; and (b) the direct excitation of the azide moiety leading to the formation of ${ }^{1} \mathrm{DPP}-\mathrm{N}$, along with $\mathrm{N}_{2}$ extrusion. Our femtosecond spectroscopic results favored the mechanism involving energy transfer.

\section{Experimental and Computational Details}

\section{III.A. Computational Methods}


All calculations were performed with the Turbomole- $5.80^{14}$ suite of programs at the Ohio Supercomputer Center. The ground state geometries were optimized with second-order coupled cluster methods using the resolution-of-the-identity approximation for the electron-electron repulsion integrals (RI-CC2) ${ }^{15}$ and with Becke's three-parameter hybrid exchange functional ${ }^{16}$ in combination with Lee, Yang, and Parr's correlation functional ${ }^{17}$ (B3LYP). The triple-zeta valence polarized (TZVP) basis sets ([14s9p1d]/[5s4p1d] for P; [11s6p1d]/[5s3p1d] for C, N, and $\mathrm{O}$; and $[5 \mathrm{~s} 1 \mathrm{p}] /[3 \mathrm{~s} / 1 \mathrm{p}]$ for $\mathrm{H}$ ), as developed by Ahlrichs and co-workers, ${ }^{18}$ were used for all of these calculations. To calculate the vertical excitations and to obtain the difference density plots, the time-dependent B3LYP (TD-B3LYP) methodology ${ }^{23}$ was used as well as the RI-CC2 methodology. ${ }^{15}$ All of the stationary points were confirmed to be minima by calculating vibrational frequencies analytically with the AOFORCE module, as implemented in Turbomole.

\section{III.B. Femtosecond Broadband UV-Vis Transient Absorption Spectrometer}

The measurements discussed in the paper were performed using the nanosecond and femtosecond time-resolved absorption spectrometers described elsewhere. ${ }^{21,19}$ The excitation wavelength used in the ultrafast experiments was $260 \mathrm{~nm}$. (The nanosecond LFP measurements were carried out using $266 \mathrm{~nm}$ excitation, while $254 \mathrm{~nm}$ light source was used for preparativescale product analyses.) The concentrations of the photolytic precursors were adjusted in each case so that all samples used for spectroscopic studies would have an absorption of $\sim 1.0$ at 260 $\mathrm{nm}$. All of the curve fittings were performed with the Igor Pro 6.0 software.

\section{III.C. Materials}

Diphenylphosphoryl azide (DPP- $\mathrm{N}_{3}$ ) was used as purchased from Aldrich. Spectroscopic grade acetonitrile was used as purchased from Fisher Scientific Inc. DPP- $\mathrm{NH}_{2}$ was synthesized according to the procedure published in the literature. ${ }^{20}$ 


\section{Results and Discussion}

\section{IV.A Computational Chemistry: Ground state geometries and vertical excitations}

Optimized geometries of DPP-N $\mathrm{N}_{3}$ at the RI-CC2/TZVP and B3LYP/TZVP levels of theory are shown in Figure 1. The view shown in the figure is along the $\mathrm{O}=\mathrm{P}$ bond, as viewed from oxygen to phosphorus. Both of the phenyl rings are in a 'geared' conformation and each ring has a $50-60^{\circ}$ torsion angle with respect to the $\mathrm{O}=\mathrm{P}$ bond. The azide unit is found to be almost parallel to the $\mathrm{O}=\mathrm{P}$ bond and has a typical geometry, as described in the literature. ${ }^{21,22}$ Using a coupled cluster ${ }^{15}$ and a time-dependent density functional theory (TD-DFT) ${ }^{23}$ approach, we calculated the vertical excitations with these optimized geometries for the $\mathrm{S}_{0}$ ground state. (Table 1) The RI-CC2/TZVP level of theory predicts that $\mathrm{S}_{1}$ and $\mathrm{S}_{2}$ states are degenerate, whereas TD-B3LYP/TZVP calculations separate these two excited singlet states by a small energetic difference. However, the energy difference between the $\mathrm{S}_{1}$ and $\mathrm{S}_{2}$ states is very small, and both of these states have low oscillator strengths. Each of these Frank-Condon states is a combination of many orbital-to-orbital transitions; therefore, a single orbital-to-orbital transition cannot be assigned. Consequently, it is very difficult to predict the photochemical behavior of a particular excited state by visualizing the molecular orbitals involved in the formation of that singlet excited state.

Table 1. Calculated vertical excitations for diphenylphosphoryl azide (DPP-N $)^{a}{ }^{\text {a }}$

\begin{tabular}{ccccc}
\hline & \multicolumn{2}{c}{ RI-CC2/TZVP } & \multicolumn{2}{c}{ TD-B3LYP/TZVP } \\
\hline $\begin{array}{c}\text { Excited } \\
\text { State }\end{array}$ & $\begin{array}{c}\text { Wavelength } \\
\text { (nm) }\end{array}$ & $\begin{array}{c}\text { Oscillator } \\
\text { Strength }\end{array}$ & $\begin{array}{c}\text { Wavelength } \\
\text { (nm) }\end{array}$ & $\begin{array}{c}\text { Oscillator } \\
\text { Strength }\end{array}$ \\
\hline $\mathrm{S}_{1}$ & 237 & $1.0 \times 10^{-3}$ & 249 & $1.8 \times 10^{-2}$ \\
$\mathrm{~S}_{2}$ & 237 & $1.0 \times 10^{-3}$ & 242 & $6.2 \times 10^{-3}$ \\
$\mathrm{~S}_{3}$ & 230 & $3.7 \times 10^{-4}$ & 238 & $5.3 \times 10^{-3}$ \\
$\mathrm{~S}_{4}$ & 205 & $3.0 \times 10^{-2}$ & 233 & $8.8 \times 10^{-3}$ \\
\hline
\end{tabular}

a The optimized geometry for the $\mathrm{S}_{0}$ ground state of DPP- $\mathrm{N}_{3}$ was computed at the same level of theory as the excitation energies. 

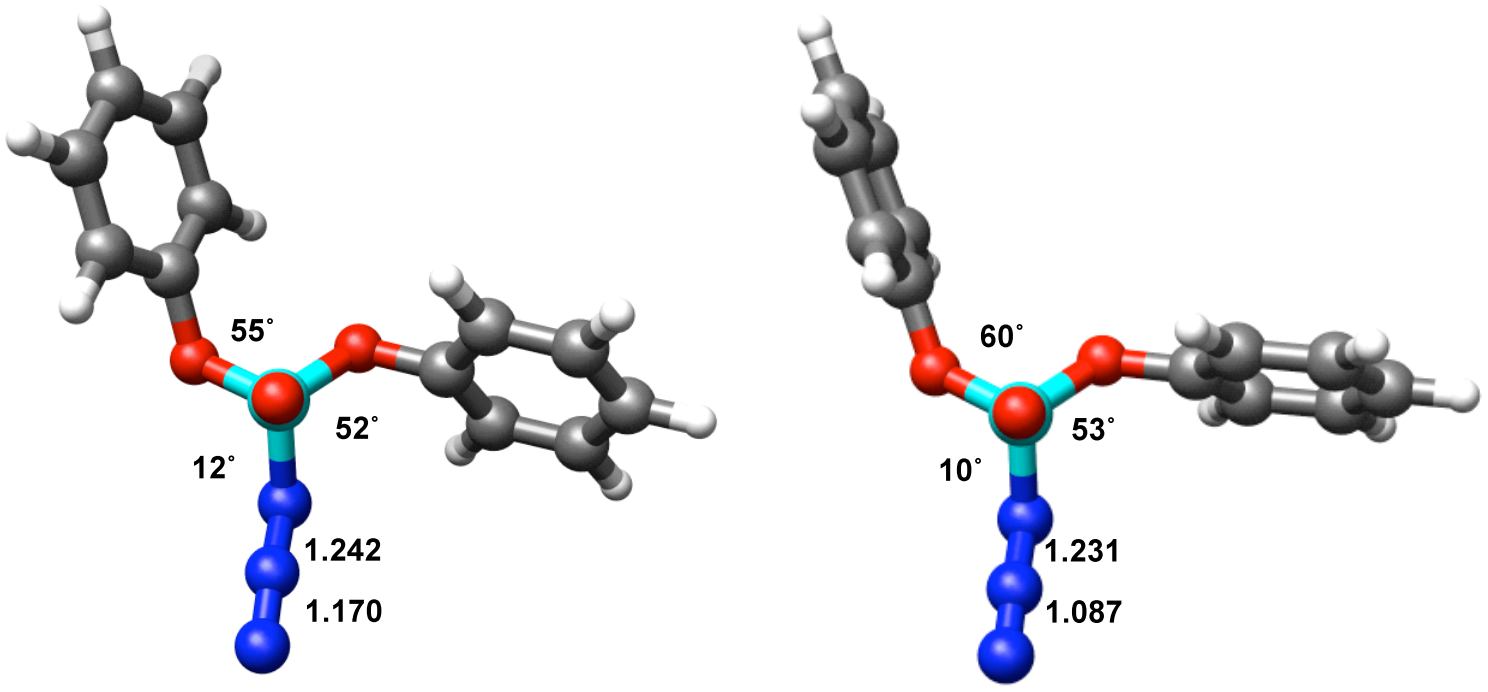

Figure 1. Optimized geometries of diphenylphosphoryl azide $\left(\mathrm{DPP}-\mathrm{N}_{3}\right)$ at the RI-CC2/TZVP (left) and TD-B3LYP/TZVP (right) levels of theory. (Selected distances are shown in $\AA$, and dihedral angles are measured with respect to the $\mathrm{O}=\mathrm{P}$ bond.)

\section{IV.B Computational Chemistry: Difference density plots}

To address the challenge of the multi-configurational nature of the excited states, we obtained electron density difference plots for the four lowest energy singlet excited states of $\mathrm{DPP}-\mathrm{N}_{3}$. We have recently applied this methodology for different photochemical problems such as predicting the photochemistry of aryl azides, ${ }^{21,22}$ naphthylimides, ${ }^{24}$ and inorganic complexes, ${ }^{25}$ investigating the photochemical mechanism of nitro-substituted polycyclic aromatic hydrocarbons, ${ }^{26}$ and predicting the character of the excited states of N-confused porphyrins. ${ }^{27}$ This strategy has also been used earlier with other $a b$ initio methods. ${ }^{28}$ To obtain the difference density plots, we calculated the electronic density for the Frank-Condon singlet excited state, followed by subtraction of the ground state's electron density at the same geometry. Such plots show the overall change in the electron density upon vertical excitation wherein the green contours depict the accumulation of electron density in the excited state and the red contours illustrate the loss of electron density from the $\mathrm{S}_{0}$ ground state at some contour value. 

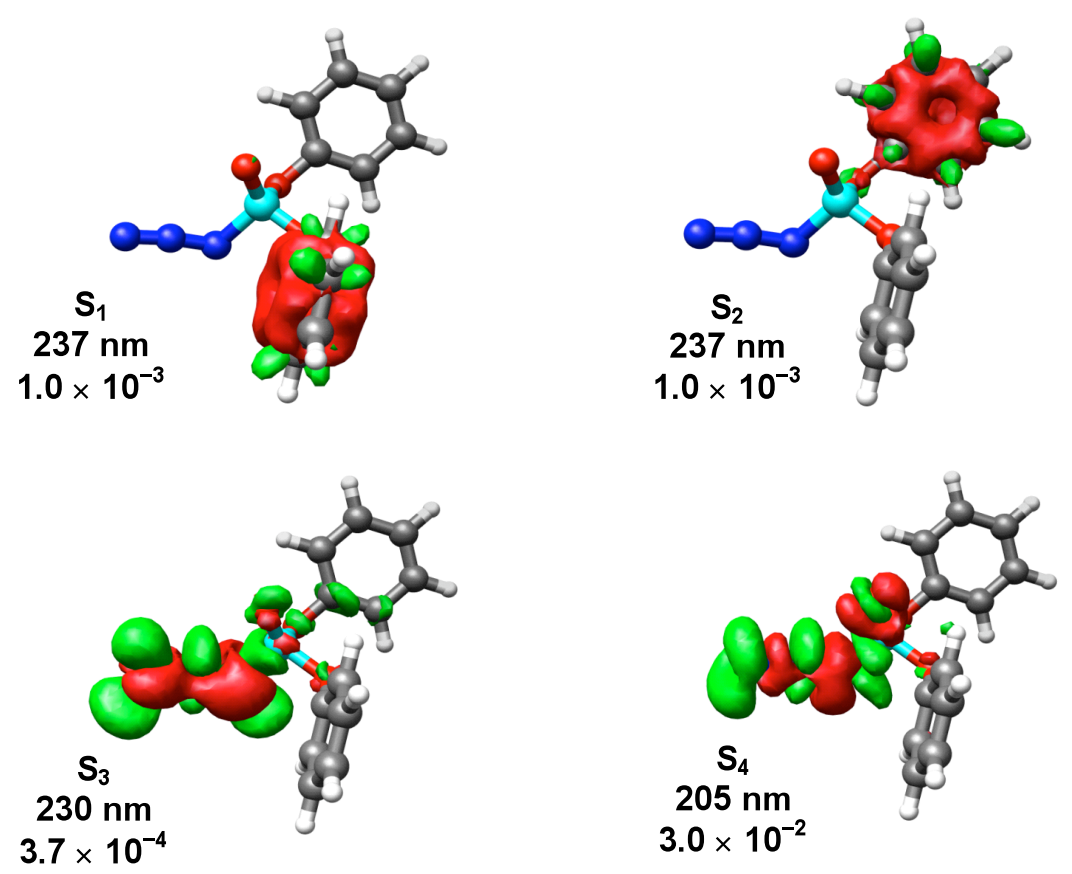

Figure 2. Difference density plots for $\mathrm{S}_{\mathrm{n}}(\mathrm{n}=1$ to 4$)$ of DPP- $\mathrm{N}_{3}$ at the RI-CC2/TZVP level of theory. The energy of the excitation is provided as well as the oscillator strength. The green contours depict the accumulation of electron density in the excited state, and the red contours illustrate the loss of electron density from the $\mathrm{S}_{0}$ ground state. The isocontour values are -0.005 and +0.0005 au.
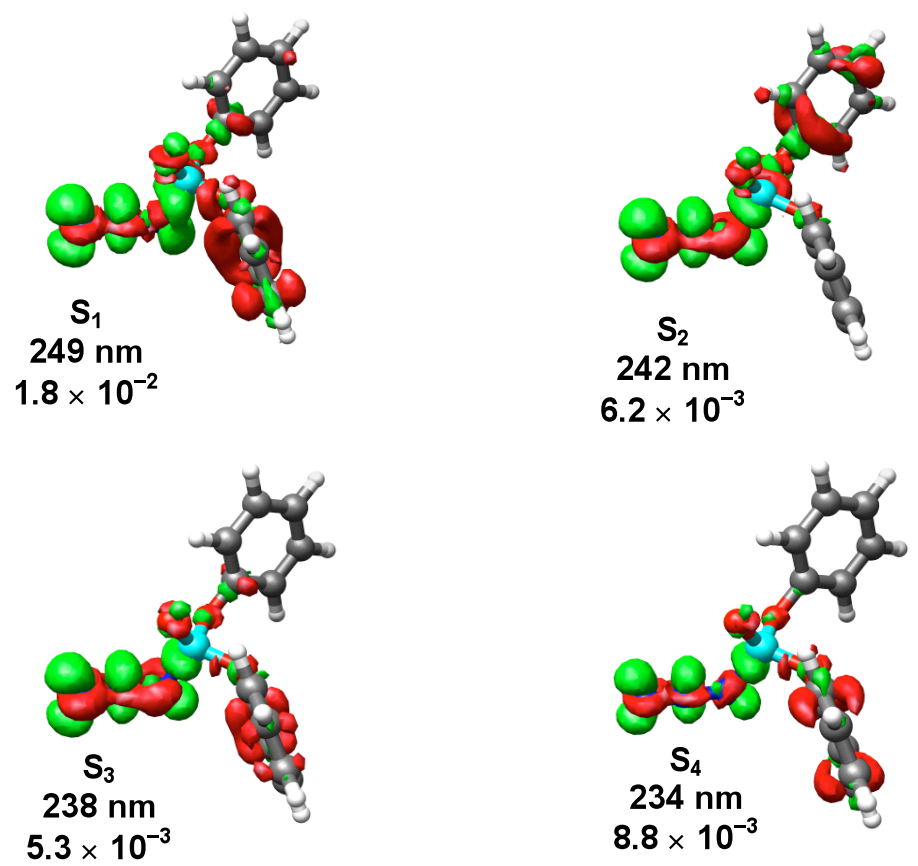

$6.2 \times 10^{-3}$

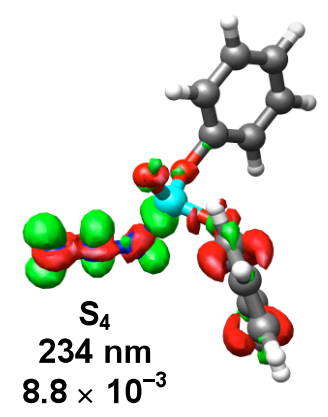

Figure 3. Difference density plots for $\mathrm{S}_{\mathrm{n}}(\mathrm{n}=1$ to 4$)$ of DPP- $\mathrm{N}_{3}$ at the TD-B3LYP/TZVP level of theory. The energy of the excitation is provided as well as the oscillator strength. The green contours depict the accumulation of electron density in the excited state, and the red contours illustrate the loss of electron density from the $\mathrm{S}_{0}$ ground state. The isocontour values are \pm 0.003 
For DPP- $\mathrm{N}_{3}$, the calculated difference density plots are shown in Figures 2 and 3 at the RI-CC2/TZVP and TD-B3LYP/TZVP levels of theory, respectively. Interestingly, the calculated difference density plots for the $S_{1}$ and $S_{2}$ states by the two different theoretical methods show different photochemical consequences. The RI-CC2 calculations predicted the $\mathrm{S}_{1}$ and $S_{2}$ states to possess $\left(\pi, \pi^{*}\right)$ character localized on the phenyl rings. On the other hand, the TD-B3LYP method predicted that the $\mathrm{S}_{1}$ and $\mathrm{S}_{2}$ states are primarily localized on the azide group having $\left(\pi, \pi^{*}\right.$ in-plane $)$ character, which was also reported previously21,22 for various aryl azides.

Furthermore, the difference density plots for $S_{3}$ and $S_{4}$ showed $\left(\pi, \pi^{*}\right.$ in-plane $)$ characteristics (of the azide moiety) at the RI-CC2 level and $\left(\pi, \pi^{*}\right.$ ) characteristics (of the phenyl rings) at the TD-B3LYP level. The dramatic changes involving the azide unit of these states would suggest that the proximal $\mathrm{N}-\mathrm{N}$ bond would be weakened upon excitation to these excited states, and thus lead to phosphorylnitrene formation after extrusion of molecular nitrogen. However, the computational results suggested two different pathways for ${ }^{1} \mathrm{DPP}-\mathrm{N}$ formation as shown in Scheme 2. While TD-B3LYP predicted direct formation of ${ }^{1} \mathrm{DPP}-\mathrm{N}$ via the azide excited state, the RI-CC2 results suggested energy transfer from the phenyl $\left(\pi, \pi^{*}\right)$ excited state to eventually form the ${ }^{1} \mathrm{DPP}-\mathrm{N}$.

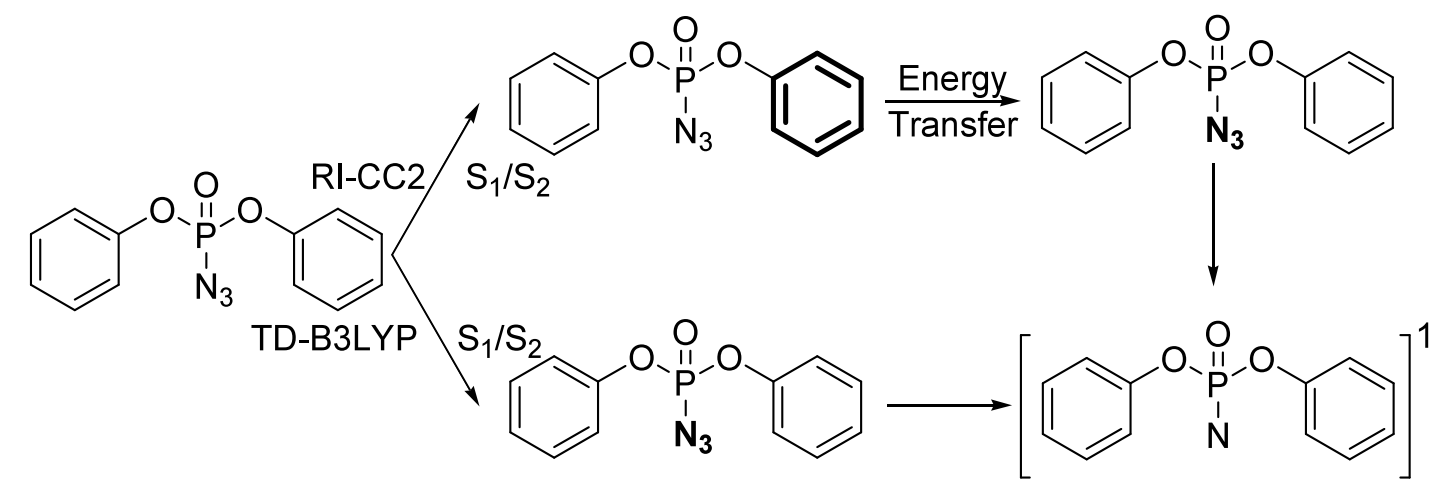

Scheme 2. The photochemistry of DPP- $\mathrm{N}_{3}$ as predicted by the RI-CC2/TZVP (top) and TDB3LYP/TZVP (bottom) levels of theory. The initially photoexcited unit of the molecule is depicted in bold. 
To our knowledge, ${ }^{21,22}$ such a discrepancy between these two level of theories has not been previously noted for azides. Both theoretical methods provided remarkably similar predictions in the past that were comparable to the experimental observations. ${ }^{21,22,26,27}$ However, the preceding computations were performed on aryl azides, for which the lowest excited singlet state always had $\left(\pi, \pi^{*}\right.$ in-plane $)$ characteristics of the azide moiety, thereby leading to singlet nitrene formation. Based on these computational results, we predict that photolysis of DPP- $\mathrm{N}_{3}$ at about $250 \mathrm{~nm}$ will result in pumping the precursor molecule to either a singlet azide excited state or a singlet $\left(\pi, \pi^{*}\right)$ excited state of the phenyl ring, which will then transfer its energy to the azide unit. In either case, the singlet nitrene will then be formed, which may be observed using ultrafast absorption spectroscopic measurements.

\section{IV.C Ultrafast spectroscopic studies of diphenylphosphoryl azide}

The singlet state of diphenylphosphorylnitrene $\left({ }^{1} \mathrm{DPP}-\mathrm{N}\right)$ has never been observed previously, although triplet phosphorylnitrenes have been observed by laser flash photolysis (LFP) and electron paramagnetic resonance (EPR) methods. ${ }^{11,12}$ In particular, ${ }^{3} \mathrm{DPP}-\mathrm{N}$ was found to have lifetimes on the order of microseconds, ${ }^{12}$ therefore, they are not observed in our current ultrafast experiments. The results of ultrafast time-resolved absorption spectroscopy experiments for DPP- $\mathrm{N}_{3}$ are shown in Figure 4 after $260 \mathrm{~nm}$ photolysis of a $\sim 16-18 \mathrm{mM}$ solution of the azide in acetonitrile. At ambient temperature, the excited state of DPP- $\mathrm{N}_{3}$, species A, is observed at about $475 \mathrm{~nm}$ (Figure 4a). This excited state is observed immediately after the sample is exposed to the laser pulse. Based on our calculations, this species could be assigned either to the azide $\left(\pi, \pi^{*}\right.$ in-plane $)$ excited state or a $\left(\pi, \pi^{*}\right)$ excited state of the phenyl rings of DPP- $\mathrm{N}_{3}$ (Figures 2 and 3). The decay of this species is observed at about 5 ps after photolysis and a new species (B) then grows in at $525 \mathrm{~nm}$ over $150 \mathrm{ps}$ (Figure $4 \mathrm{~b}$ ); species $\mathbf{B}$ 
then decays after 150 ps and almost completely disappears at about 3000 ps (Figure 4c) after the initial laser pulse. It is possible for the species $\mathbf{B}$ to be the ylide as a molecule of acetonitrile coordinates to the singlet phosphorylnitrene. However, similar experiments conducted in methanol show the same transients with approximately same lifetimes, thus we can rule out the possibility of $\mathbf{B}$ being the ylide. (See supporting information) We propose that species $\mathbf{B}$ is the singlet diphenylphosphorylnitrene, ${ }^{1} \mathrm{DPP}-\mathrm{N}$. It is important to note that vibrational cooling or band narrowing was not observed for the $525 \mathrm{~nm}$ band; the time constant for evolution of the 475 $\mathrm{nm}$ band is about $20-30$ ps which is on the same time scale for which vibrational cooling typically occurs. Furthermore, a small absorption seems to be growing at about $350 \mathrm{~nm}$ in Figure $4 \mathrm{~b}$; however, any band above $360 \mathrm{~nm}$ is unreliable in the presented ultrafast data due to very high-signal-to-noise ratio.

Since the predicted singlet nitrene and the second short-lived, promptly formed species absorbing at $475 \mathrm{~nm}$ have an overlapping absorption band, we performed global fitting of the data to calculate the lifetimes of the intermediates. De-convolution of the data was achieved by fitting to the expression:

$$
f(x)=A_{1} \exp \left(-x / t_{1}\right)+A_{2} \exp \left(-x / t_{2}\right)+A_{3}
$$

in which the first and second terms correspond to the excited state of the molecule and the singlet nitrene, respectively, and the third term is the offset (residual absorption) remaining after 3 ns. The global fitting procedure yielded the lifetime of the excited state of DPP $-\mathrm{N}_{3}$ to be $28 \pm 9$ ps (species A), and that the lifetime of the ${ }^{1} \mathrm{DPP}-\mathrm{N}$ is about $480 \pm 50$ ps (species B). 


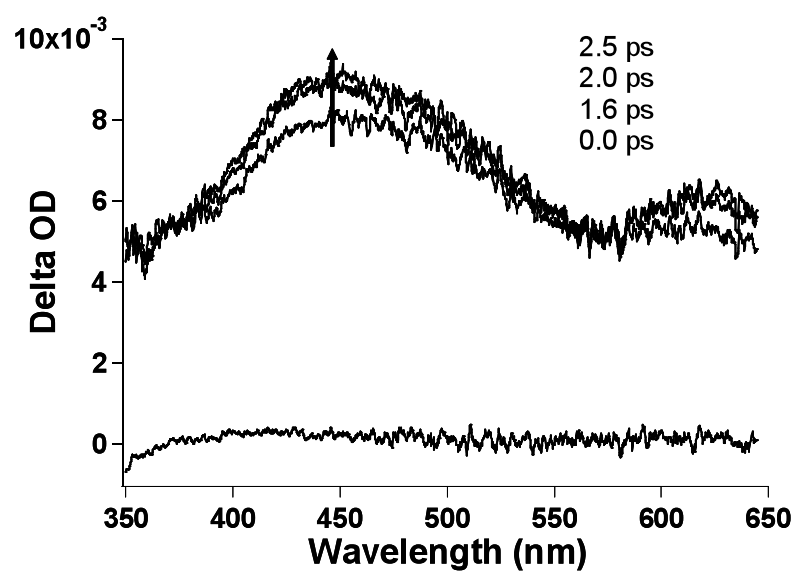

(a)

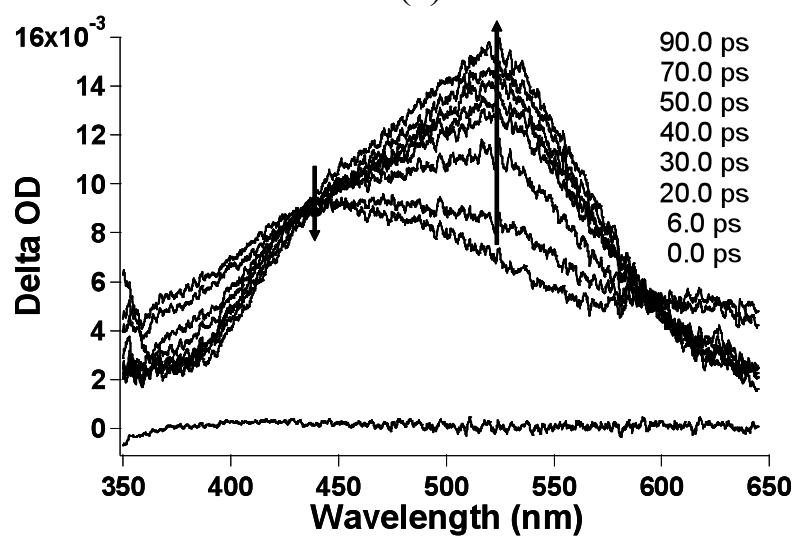

(b)

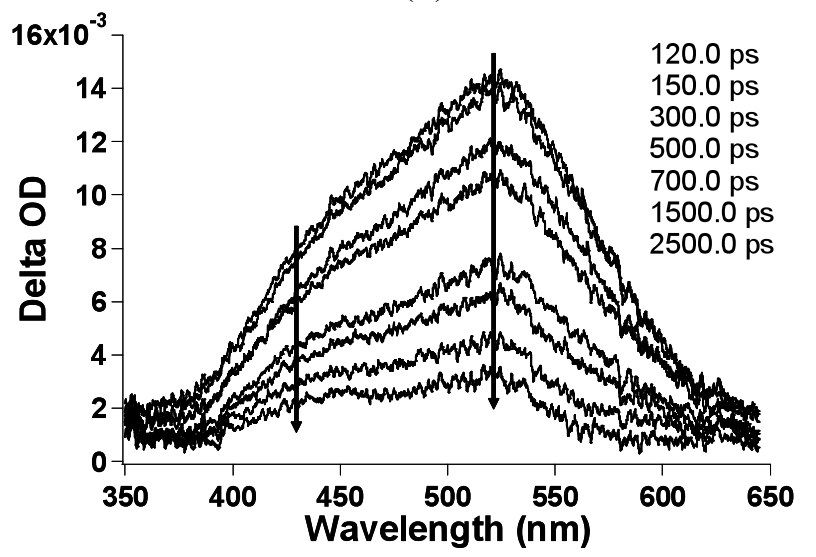

(c)

Figure 4. Femtosecond UV-Vis transient absorption spectra obtained with $260 \mathrm{~nm}$ pulsed photolysis of $\sim 16-18 \mathrm{mM}$ DPP- $\mathrm{N}_{3}$ in acetonitrile: (a) evolution of a $\left(\pi, \pi^{*}\right)$ excited state, intermediate $\mathbf{A}$; (b) depletion of the $\left(\pi, \pi^{*}\right)$ intermediate and evolution of ${ }^{1} \mathrm{DPP}-\mathrm{N}$, intermediate B; (c) decay of ${ }^{1} \mathrm{DPP}-\mathrm{N}$. 


\section{IV.D Ultrafast time-resolved spectroscopic studies on quenching of the singlet nitrene}

Tris-(trimethylsilyl)silane, $\left(\left(\mathrm{H}_{3} \mathrm{C}\right)_{3} \mathrm{Si}\right)_{3} \mathrm{Si}-\mathrm{H}$ (TRIS), is known to quench singlet nitrenes, presumably by insertion into the $\mathrm{Si}-\mathrm{H}$ bond. ${ }^{12}$ In order to verify that the relatively long-lived $(\sim 0.5 \mathrm{~ns})$ species in our ultrafast $\mathrm{UV}-\mathrm{V}$ is experiments is ${ }^{1} \mathrm{DPP}-\mathrm{N}$, we performed ultrafast timeresolved experiments in the presence of various concentrations of the silane. Similar to the experiments discussed above, two species were observed at 475 and $525 \mathrm{~nm}$, respectively. However, the lifetime of the $525 \mathrm{~nm}$ species was observed to decrease with the increasing concentration of TRIS. Upon single exponential fitting of the data at $550 \mathrm{~nm}$, we obtained the lifetimes ( $t$ ) of the second species at different concentrations of TRIS. A plot of $1 / \tau$ versus TRIS concentration is roughly linear (Figure 5). The slope of this plot is $1.13 \times 10^{10} \mathrm{M}^{-1} \mathrm{~s}^{-1}$, which is the absolute, second-order rate constant for reaction of the nitrene with the silane. The $y(1 / \tau)$ intercept corresponds to a value of $\tau \sim 900 \mathrm{ps}$. This lifetime determined from the intercept is larger than the $480 \pm 50$ ps measured by a direct measurement of the decay in the absence of silane. As there is a greater error in the extrapolation to the y intercept, the directly measured value is deemed more accurate. Gohar and Platz assumed that the second-order rate constant for reaction of the nitrene with silane was $5.0 \times 10^{9} \mathrm{M}^{-1} \mathrm{~s}^{-1}$ and on the basis of this assumption, also deduced that the singlet nitrene lifetime was $1 \mathrm{~ns}$ in 1,2 -dichloroethane and methanol. ${ }^{12}$ This is in reasonable agreement with the results of this work and further supports our assignment of species B as ${ }^{1}$ DPP-N. Moreover, if we recalculate the Gohar and Platz lifetime using a second-

order rate constant of $1 \times 10^{10} \mathrm{M}^{-1} \mathrm{~s}^{-1}$, their data predict a lifetime of $500 \mathrm{ps}$, in excellent accord with this study. 


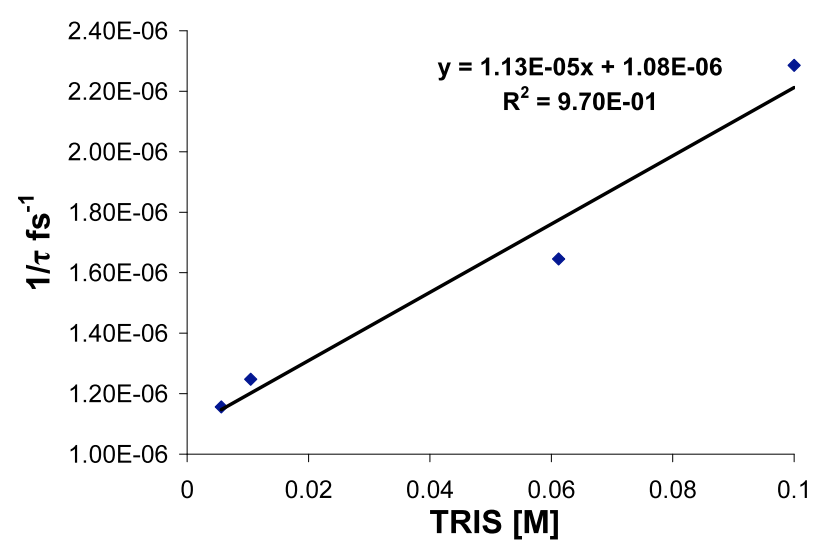

Figure 5. A plot of the observed pseudo-first-order rate constant $(1 / \tau)$ of decay of singlet nitrene versus tris-(trimethylsilyl)silane concentration. The nitrene was produced after $260 \mathrm{~nm}$ excitation of DPP $-\mathrm{N}_{3}$. A single exponential equation was fit to the data obtained from kinetic traces monitored at $550 \mathrm{~nm}$ after photolysis.

\section{IV.E Ultrafast spectroscopic studies of diphenyl phosphoramidate}

The short-lived species (28 $\pm 9 \mathrm{ps})$ observed at $475 \mathrm{~nm}$ in our ultrafast experiments can, in principle, be assigned to either the $\left(\pi, \pi^{*}\right.$ in-plane $)$ excited state localized on the azide unit or a $\left(\pi, \pi^{*}\right)$ excited state localized at the phenyl rings as predicted by our calculations. In order to rule out one of the two possibilities, we performed similar calculations and ultrafast UV-Vis experiments on diphenylphosphoramidate $\left(\mathrm{DPP}-\mathrm{NH}_{2}\right)$, in which the azide group of $\mathrm{DPP}-\mathrm{N}_{3}$ is replaced by an amine moiety.

The geometries of $\mathrm{DPP}-\mathrm{NH}_{2}$ are similar to that of $\mathrm{DPP}-\mathrm{N}_{3}$ at both levels of theory (supporting information). The calculated electron density difference plots for the $\mathrm{S}_{1}$ and $\mathrm{S}_{2}$ states at the RI-CC2/TZVP and TD-B3LYP/TZVP levels of theory are shown in Figure 6. Both the $\mathrm{S}_{1}$ and $\mathrm{S}_{2}$ states are localized on one of the phenyl rings, and with a $\left(\pi, \pi^{*}\right)$ character. These plots indicated that the ultrafast $\mathrm{UV}-\mathrm{Vis}$ measurements for $\mathrm{DPP}-\mathrm{NH}_{2}$ will probably show either a short-lived singlet excited state localized on the phenyl rings if the intersystem crossing rate is very fast or a long-lived species if the intersystem crossing rate is slow. Consequently, the 
observed absorption, and its lifetime, will rule out either azide excited state formation or a ( $\pi$, $\left.\pi^{*}\right)$ excited state of the phenyl ring in the case of the DPP $-\mathrm{N}_{3}$.
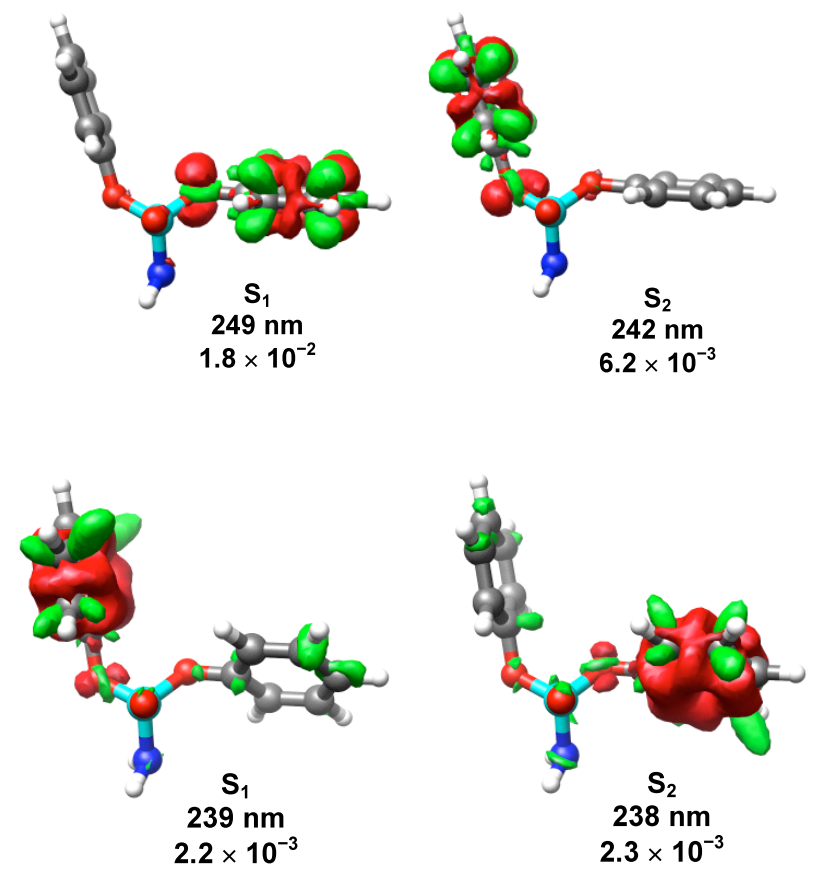

Figure 6. Difference density plots for the $\mathrm{S}_{1}$ and $\mathrm{S}_{2}$ states of DPP- $\mathrm{NH}_{2}$ at the TD-B3LYP/TZVP (top) and RI-CC2/TZVP (bottom) level of theory. The green contours depict the accumulation of electron density in the excited state, and the red contours illustrate the loss of electron density from the $\mathrm{S}_{0}$ ground state. The isocontour values are -0.005 and +0.0001 au (RI-CC2/TZVP) and \pm 0.001 au (TD-B3LYP/TZVP).

Ultrafast transient absorption experiments were performed in acetonitrile with an absorption of $\sim 1.0$ at $260 \mathrm{~nm}$, and the results are shown in Figure 7. We detected the growth of a species at $490 \mathrm{~nm}$ immediately after the first laser pulse. This intermediate A decayed after about $7 \mathrm{ps}$, and a new species $\mathbf{B}$ was found to grow as a broad peak at $550 \mathrm{~nm}$. The intermediate B was formed over $500 \mathrm{ps}$, followed by an extremely slow decay. Since, the decay of intermediate $\mathbf{B}$ is very slow, its lifetime could not be determined by ultrafast spectroscopy. We estimate the lifetime of $\mathbf{B}$ to be on the order of several nanoseconds. The intermediate $\mathbf{A}$ is predicted to be a singlet $\left(\pi, \pi^{*}\right)$ excited state localized on the phenyl ring, a result that is 
consistent with the computational results. This singlet excited state rapidly intersystem crosses to form a triplet, presumably a $\left(\pi, \pi^{*}\right)$, excited state, as intermediate $\mathbf{B}$ has no obvious competing photochemical pathway. While studying the ultrafast photolysis of aryl azides, Platz and coworkers ${ }^{21}$ observed a similar singlet $\left(\pi, \pi^{*}\right)$ excited state localized on the aryl ring at a similar wavelength, $\sim 480 \mathrm{~nm}$.

Since there is an overlap region for $\mathbf{A}$ and $\mathbf{B}$ in the transient spectra, global fitting was attempted to de-convolute the areas under the two overlapping curves. However, the growth of B completely obscured the spectral region corresponding to A. Moreover, B has almost no decay within the time scale used in the experiments; therefore, a global fit would not yield results with reliable accuracy. However, as $\mathbf{A}$ decayed, the second species $\mathbf{B}$ rose suggesting that the excited state of DPP- $\mathrm{NH}_{2}$ (i.e., A) transforms into species B. Therefore, the growth lifetime of B should correspond to the lifetime of intermediate A. Thus, we monitored the kinetic traces at $550,560,570,580,590,600$ and $610 \mathrm{~nm}$ at which the spectral signature of $\mathbf{A}$ is negligible in order to monitor the formation of $\mathbf{B}$. The kinetic traces from 0 to $500 \mathrm{ps}$ were fitted to a single exponential function to calculate the growth of the intermediate B. For $\mathrm{DPP}-\mathrm{NH}_{2}$, the average $\tau_{\mathbf{B}}^{\text {rise }}$ was determined to be $33 \pm 8 \mathrm{ps}$, which is also the lifetime of the intermediate $\mathbf{A}$. This lifetime is comparable to, but slightly longer than, the deduced lifetime for the singlet excited state of DPP $-\mathrm{N}_{3}$ (Figure 4, intermediate A) obtained by global fitting ( $\left.28 \pm 9 \mathrm{ps}\right)$, as predicted by the mechanistic scheme. Global fitting analysis of the ultrafast experimental data of $\mathrm{DPP}-\mathrm{NH}_{2}$ established the lifetime of species $\mathbf{A}$ to be about 100 ps (although with a large error), This species, attributed to the singlet excited state localized on the phenyl ring of $\mathrm{DPP}-\mathrm{NH}_{2}$, has a longer lifetime than the corresponding singlet exited phenyl ring observed for DPP- $\mathrm{N}_{3}$ at 475 $\mathrm{nm}$. The relatively longer lifetime of the phenyl excited state of $\mathrm{DPP}-\mathrm{NH}_{2}$ is reasonable since 
${ }^{1} \mathrm{DPP}-\mathrm{NH}_{2}$ (or species $\mathbf{A}$ ) can only decay through intersystem crossing, while the singlet excited state of DPP- $\mathrm{N}_{3}$ has several other possibilities, including energy transfer to the azide moiety.

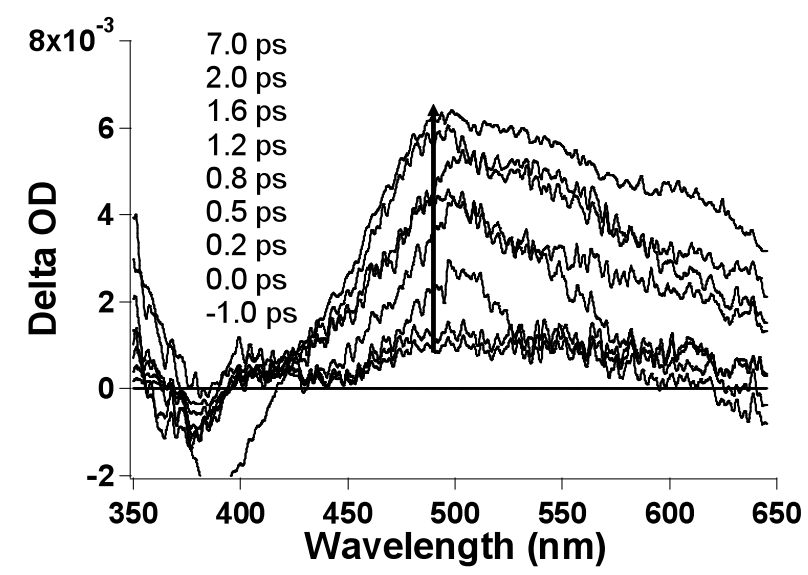

(a)

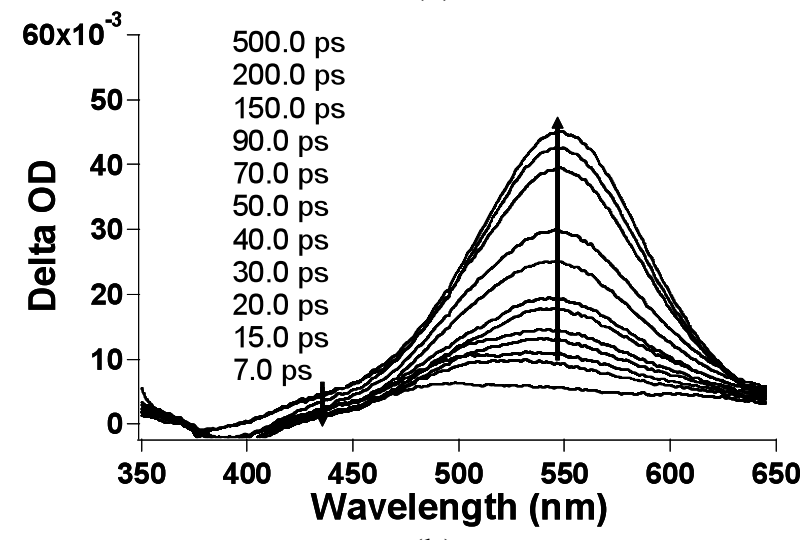

(b)

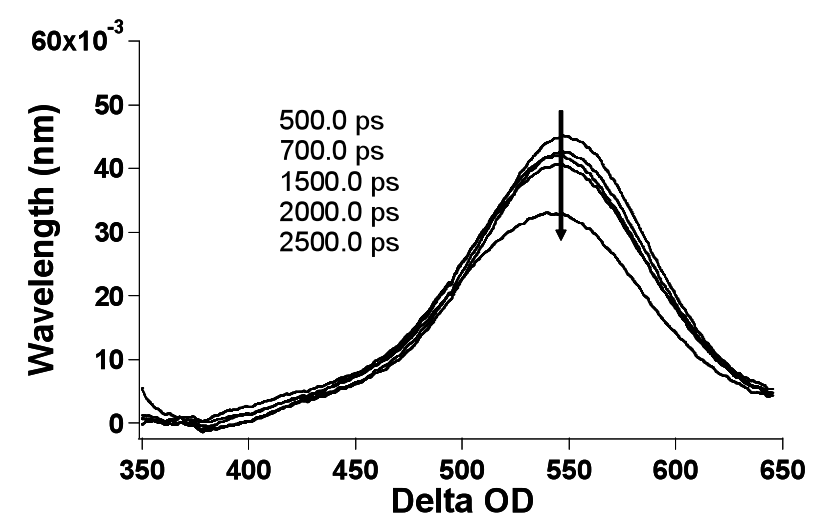

(c)

Figure 7. Femtosecond UV-Vis transient absorption spectra produced by $260 \mathrm{~nm}$ photolysis of $\sim 12 \mathrm{mM}$ DPP $-\mathrm{NH}_{2}$ in acetonitrile: (a) evolution of a $\left(\pi, \pi^{*}\right)$ excited state, intermediate $\mathbf{A}$; (b) 
depletion of the $\left(\pi, \pi^{*}\right)$ excited state and evolution of triplet $\left(\pi, \pi^{*}\right)$ excited state, intermediate $\mathbf{B}$, and (c) decay of the triplet intermediate.

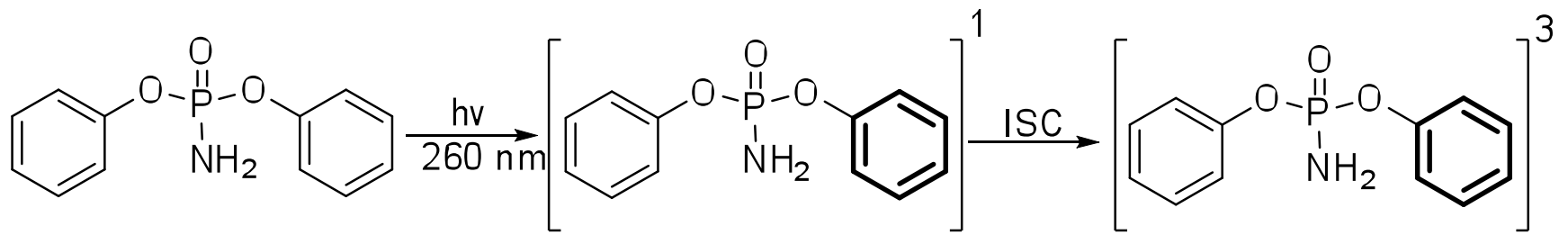

Scheme 3. Ultrafast UV-Vis photochemistry of diphenylphosphoramidate. Note that the lowest energy excited state is localized on the aromatic moiety of this compound.

Thus, from the DPP- $\mathrm{NH}_{2}$ spectral analysis, we conclude that the short-lived intermediate observed in the ultrafast UV-Vis absorption spectra of DPP- $\mathrm{N}_{3}$ is a $\left(\pi, \pi^{*}\right.$ ) excited state (of the phenyl ring) rather than the $\left(\pi, \pi^{*}\right.$ in-plane) excited state (of the azide group). Inspection of the geometry of DPP- $\mathrm{N}_{3}$ (Figure 1) showed that the phenyl rings were oriented towards the azide group, thereby favoring fast energy transfer. Since DPP- $\mathrm{NH}_{2}$ does not have a competing energy transfer decay pathway (such as to the azide group of $\mathrm{DPP}-\mathrm{N}_{3}$ ), we posit that the excited state of $\mathrm{DPP}-\mathrm{NH}_{2}$ should be longer lived than the DPP-N $\mathrm{N}_{3}$ excited state, which is in good agreement with the aforementioned observations. The slower, competing decay pathway of $\mathrm{DPP}-\mathrm{NH}_{2}$ is intersystem crossing to the triplet manifold. Thus, we assign species $\mathbf{B}$ in $\mathrm{DPP}-\mathrm{NH}_{2}$ to a triplet state, a species, which is formed from the (A) singlet $\left(\pi, \pi^{*}\right)$ phenyl excited state with a time constant of $33 \pm 8$ ps. This conclusion was confirmed by nanosecond LFP studies with DPP-NH $\mathrm{NH}_{2}$ in which the $550 \mathrm{~nm}$ band was again observed, and which had a lifetime of $8.5 \pm 0.5 \mathrm{~ns}$. The lifetime of this transient species was sensitive to oxygen concentration and found to have a shorter lifetime $(\sim 5 \mathrm{~ns})$ and lower intensity, consistent with its assignment as a triplet species. These results also confirmed that the RI-CC2/TZVP calculations predicted the correct 
photochemistry for DPP- $\mathrm{N}_{3}$, whereas the TD-B3LYP/TZVP calculations were less consistent with experiment in this system.

In our ultrafast experiments with DPP- $\mathrm{N}_{3}$, similar to the ultrafast studies on aryl azides, ${ }^{21}$ we could not observe the azide dissociative state which can be formed following energy transfer from the initially generated $\left(\pi, \pi^{*}\right)$ singlet excited state of the phenyl ring. Furthermore, vibrational cooling (band narrowing) of the $525 \mathrm{~nm}$ absorption was not observed. In the case of

naphthyl azide, ${ }^{21}$ the azide dissociative state, which was not observed, was predicted to have a lifetime shorter than $1 \mathrm{ps}$. In general, the barrier for the loss of molecular nitrogen from the azide dissociative state is very small $(<1-2 \mathrm{kcal} / \mathrm{mol}){ }^{21}$ hence, nitrene formation can be expected to occur almost instantaneously upon populating the dissociative excited state of the azide. The rate of growth of the $525 \mathrm{~nm}$ band was the same as the rate of decay of the $475 \mathrm{~nm}$ band, which is about 20 ps. As these time scales are similar to that of vibrational cooling, we are not surprised that we did not observe any band-narrowing at $525 \mathrm{~nm}$.

\section{IV.F Chemical Analysis of Reaction Products}

As the photoproducts of DPP-N $\mathrm{N}_{3}$ have not been completely characterized, we decided to isolate the photoproducts following preparative scale photolysis of DPP- $\mathrm{N}_{3}$, using acetonitrile and cyclohexane as solvents.

Steady-state photolysis of DPP-N $\mathrm{N}_{3}$ in acetonitrile using a high-pressure mercury lamp primarily formed an un-characterizable brown polymer presumably due to several reactions including ylide formation, nitrene-dimerization, and hydrogen-atom abstraction reactions. Nevertheless, analysis of the mixture of volatile products in acetonitrile and cyclohexane by GCMS and ESI-MS confirmed the singlet nitrene insertion into the solvent. In order to understand if the product formation was due to secondary photochemistry, irradiated DPP- $\mathrm{N}_{3}$ was dissolved 
in acetonitrile and photolyzed at $260 \mathrm{~nm}$ using the femtosecond absorption setup for about 1-2 hour. Although the precipitates were not formed initially in the femtosecond apparatus, the solution turned yellow-brown in color and began to form a small amount of a visible precipitate over time.

We carried out the preparative scale, steady-state photolysis of DPP- $\mathrm{N}_{3}$ in cyclohexane at $>254 \mathrm{~nm}$, and isolated two products; formation of DPP-CH was observed due to the singlet nitrene insertion reaction into a $\mathrm{C}-\mathrm{H}$ bond of the solvent. The photoproduct was isolated by silica gel chromatography and characterized by ${ }^{1} \mathrm{H} \mathrm{NMR},{ }^{13} \mathrm{C} \mathrm{NMR}$, IR and MS techniques (see supporting information for experimental details). We performed similarly studies with tristrimethylsilylsilane as a quenching agent in acetonitrile. From characterization of those mixtures, we also detect a small peak in the mass spectra consistent with trapping of the nitrene with the silane (see supporting information).

All of these reactions are consistent with formation of a very reactive singlet diphenylphosphorylnitrene.

\section{Conclusions}

We investigated the photochemistry of $\mathrm{DPP}-\mathrm{N}_{3}$ by computational and experimental methods. Our computational results at the RI-CC2/TZVP and TD-B3LYP/TZVP levels of theory were not in agreement with each other; however, we do note that the excitation energies are very similar (within $30 \mathrm{~nm}$ ) for the first few excited states. The experimental results are clearly in better agreement with the RI-CC2 results. We established the formation of two transient species with a lifetime of about 28 and $480 \mathrm{ps}$, respectively, using ultrafast UV-Vis transient absorption measurements for $\mathrm{DPP}-\mathrm{N}_{3}$. These transient absorptions were assigned to the singlet excited state of the azide $\left({ }^{1} \mathrm{DPP}-\mathrm{N}_{3} *\right)$, localized on the phenyl ring, and the direct 
observation of the singlet diphenylphosphorylnitrene $\left({ }^{1} \mathrm{DPP}-\mathrm{N}\right)$, respectively. We confirmed our assignments using quenching studies by tris-trimethylsilylsilane and by the ultrafast UV-Vis analysis of DPP- $\mathrm{NH}_{2}$.

This is the first spectroscopic observation of a singlet phosphorylnitrene, and this reactive intermediate has a lifetime of $\sim 0.5 \mathrm{~ns}$ in solution at ambient temperature. The singlet phosphorylnitrene is generated by initial excitation of the $\left(\pi, \pi^{*}\right)$ phenyl ring of the azide precursor, energy transfer from the phenyl moiety to the azide unit, and subsequent extrusion of molecular nitrogen to form ${ }^{1} \mathrm{DPP}-\mathrm{N}$. From the product studies, we predict that DPP- $\mathrm{N}_{3}$ can serve as an effective reagent for photoaffinity labeling as upon photolysis it will produce a short-lived singlet nitrene that should react rapidly and indiscriminately with $\mathrm{C}-\mathrm{H}$ bonds within a nanosecond to form robust adducts. ${ }^{8,9,10}$

Further work for binding and photoaffinity labeling studies of DPP- $\mathrm{N}_{3}$ with HuPON1 is ongoing, and will provide further insight into developing a catalytic bioscavenger as a therapeutic against nerve agent poisoning.

\section{Acknowledgments}

We acknowledge financial support from the National Institutes of Health (U54NS058183). We also acknowledge the National Science Foundation for funding the femtosecond transient absorption UV-Vis apparatus. Generous computational resources from the Ohio Supercomputer Center are also gratefully acknowledged. 


\section{References}

${ }^{1}$ Bartels, C. F.; James, K.; La Du, B. N. Am. J. Hum. Genet. 1992, 50, 1104-1114.

2 (a) Main, A. R. Pharmacol. Ther. 1979, 6, 579-628. (b) Rozenbeny, T. L. Adv. Enzymol. 1975, 43, 103. (c) Quinn, D. M. Chem. Rev. 1987, 87, 955-979.

3 (a) Clement, J. G. Arch. Toxicol. 1992, 66, 143-144. (b) Lundy, P. M.; Hansen, A. S.; Hand, B. T.; Boulet, C. A. Toxicology 1992, 72, 99-105. (c) Worek, F.; Eyer, P.; Szinicz, L. Arch. Toxicol. 1998, 72, 580-587.

${ }^{4}$ (a) Raveh, L.; Grauer, E.; Grunwald, J.; Cohen, E.; Ashani, Y. Toxicol. Appl. Pharmacol. 1997, 145, 43-53. (b) Cerasoli, D. M.; Griffiths, E. M.; Doctor, B. P.; Saxena, A.; Fedorko, J. M.; Greig, N. H.; Yu, Q. S.; Huang, Y.; Wilgus, H.; Karatzas, C. N.; Koplovitz, I.; Lenz, D. E. Chem. Bio. Int. 2005, 157, 362-365.

5 (a) Josse, D.; Xie, W.; Renault, F.; Rochu, D.; Schopfer, L. M.; Masson, P.; Lockridge, O. Biochemistry 1999, 38, 2816-2825. (b) Harel, M.; Aharoni, A.; Gaidukov, L.; Brumshtein, B.; Khersonsky, O.; Meged, R.; Dvir, H.; Ravelli, R. B.; McCarthy, A.; Toker, L.; Silman, I.; Sussman, J. L.; Tawfik, D. S. Nat. Struct. Mol. Biol. 2004, 11, 412-419. (c) Khersonsky, O.; Tawfik, D. S. Biochemistry 2005, 44, 6371-6382 (d) Khersonsky, O.; Tawfik, D. S. J. Biol. Chem. 2006, 281, 7649-7656.

${ }^{6}$ (a) Yueng, D. T.; Lenz, D. E.; Cerasoli, D. M. FEBS J. 2005, 272, 2225 - 2230. (b) Yeung, D. T.; Josse, D.; Nicholson, J. D.; Khanal, A.; McAndrew, C. W.; Bahnson, B. J.; Lenz, D. E.; Cerasoli, D. M. Biochim. Biophys. Acta. 2004, 1702, $67-77$.

7 (a) Staros, J. V. Trends Biochem. Sci (Pers. Ed.) 1980, 5, 320-322. (b) BayLey, H. Photogenerated Reagent in Biochemistry and Molecular Biology; Elsevier: New York, 1983. (c) Bayley, H.; Straos, J. In Azides and Nitrenes; Reactivity and Utility; Academic Press: New York, $1984 ;$ p 434.

${ }^{8}$ Shioiri, T.; Ninomiya, K.; Yamada, S. J. Am. Chem. Soc. 1972, 94, 6203-6205.

${ }^{9}$ Breslow, R.; Feiring, A.; Herman, F. J. Am. Chem. Soc. 1974, 96, 5937-5939.

${ }^{10}$ Maslak, P. J. Am. Chem. Soc. 1989, 111, 8201-8207.

${ }^{11}$ Houser, M.; Kelley, S.; Maloney, V.; Marlow, M.; Steininger, K.; Zhou, H. J. Phys. Chem. 1995, 99, 7946-7950.

${ }^{12}$ Gohar, G. A.; Platz, M. S. J. Phys. Chem. A 2003, 107, 3704-3707.

${ }^{13}$ McCulla, R. D.; Gohar, G. A.; Hadad, C. M.; Platz, M. S. J. Org. Chem. 2007, 72, 94269438.

14 (a) Ahlrichs, R.; Bär, M.; Häser, M.; Horn, H.; Kölmel, C. Chem. Phys. Lett. 1989, 162, 165. (b) For the current version of TURBOMOLE, see http://www.turbomole.de. (c) Treutler, O.; Ahlrichs, R. J. Chem. Phys. 1995, 102, 346. 
15 (a) Hättig, C.; Weigend, F. J. Chem. Phys. 2000, 113, 5154-5161. (b) Hättig, C.; Köhn, A.; Hald, K. J. Chem. Phys. 2002, 116, 5401-5410. (c) Hättig, C. J. Chem. Phys. 2003, 118, 77517761.

${ }^{16}$ Becke, A. D. J. Chem. Phys. 1993, 98, 5648-5652.

${ }^{17}$ Lee, C.; Yang, W.; Parr, R. G. Phys. Rev. B 1988, 37, 785-789.

${ }^{18}$ Weigend, F.; Häser, F.; Patzelt, H.; Ahlrichs, R. Chem. Phys. Lett. 1998, 294, 143-152.

${ }^{19}$ (a) Gritsan, N. P.; Zhai, H. B.; Yuzawa, T.; Karweik, D.; Brooke, J.; Platz, M. S. J. Phys. Chem. A 1997, 101, 2833 - 2840. (b) Martin, C. B.; Shi, X.; Tsao, M.-L.; Karweik, D.; Brooke, J.; Hadad, C. M.; Platz, M. S. J. Phys. Chem. B 2002, 106, 10263 - 10271. (c) Tsao, M.-L.; Gritsan, N.; James, T. R.; Platz, M. S.; Hrovat, D. A.; Borden, W. T. J. Am. Chem. Soc. 2003, $125,9343-9358$.

${ }^{20}$ Rajaram, S.; Chary, K. P.; Iyengar, D. S. Synth. Commun. 2000, 30, 4495-4500.

${ }^{21}$ (a) Burdzinski, G.; Gustafson, T. L.; Hackett, J. C; Hadad, C. M.; Platz, M. S. J. Am. Chem. Soc. 2005, 127, 13764-13765. (b) Burdzinski, G.; Hackett, J. C; Wang, J.; Gustafson, T. L.; Hadad, C. M.; Platz, M. S. J. Am. Chem. Soc. 2006, 128, 13402-13411. (c) Wang, J.; Kubicki, J.; Burdzinski, G.; Hackett, J. C; Gustafson, T. L.; Hadad, C. M.; Platz, M. S. J. Org. Chem. 2007, $72,7581-7586$.

${ }^{22}$ Voskresenska, V.; Wilson, R. M.; Panov, M.; Tarnovsky, A. N.; Krause, J. A.; Vyas, S.; Winter, A. H.; Hadad, C. M. J. Am. Chem. Soc. 2009, 131, 11535-11547.

${ }^{23}$ Olivucci, M. Computational Photochemistry; Elsevier: Amsterdam, 2005; pp 92-128.

${ }^{24}$ Kucheryvavy, P.; Li, G.; Vyas, S.; Hadad, C. M.; Glusac, K. D. J. Phys. Chem. A 2009, 113, 6453-6461.

${ }^{25}$ Li, G.; Parimal, K.; Vyas, S.; Hadad, C. M.; Flood, A. H.; Glusac, K. D. J. Am. Chem. Soc. 2009, 131, 1156-11657.

${ }^{26}$ Vyas, S.; Onchoke, K. K.; Hadad, C. M.; Dutta, P. K. J. Phys. Chem. A 2009, 113, 1255812565.

${ }^{27}$ Vyas, S.; Hadad, C. M.; Modarelli, D. A. J. Phys. Chem. A 2008, 112, 6533-6549.

28 (a) Wiberg, K. B.; Hadad, C. M.; Breneman, C. M.; Laidig, K. E.; Murcko, M. A.; LePage, T. J. Science 1991, 252, 1266 - 1272. (b) Wiberg, K. B.; Hadad, C. M.; LePage, T. J.; Breneman, C. M.; Frisch, M. J. J. Phys. Chem. 1992, 96, 671 - 679. (c) Wiberg, K. B.; Hadad, C. M.; Foresman, J. B.; Chupka, W. A. J. Phys. Chem. 1992, 96, 10756 - 10768. (d) Hadad, C. M.; Foresman, J. B.; Wiberg, K. B. J. Phys. Chem. 1993, 97, 4293 - 4312. (e) Wiberg, K. B.; Hadad, C. M.; Ellison, G. B.; Foresman, J. B. J. Phys. Chem. 1993, 97, 13586 - 13597. 